\title{
CESIS
}

Electronic Working Paper Series

Paper No. 153

\section{Agglomeration Dynamics of Business Services}

\author{
Johan Klaesson ${ }^{\star}$ and Börje Johansson*
}

$\left({ }^{*} \mathrm{CESIS}\right.$ and JIBS)

October 2008 


\title{
AGGLOMERATION DYNAMICS OF BUSINESS SERVICES
}

\author{
Johan Klaesson and Börje Johansson, \\ Jönköping International Business School (JIBS) \\ Box 1026, 55111 Jönköping, Sweden, and Centre of Excellence for Science and \\ Innovation Studies, Royal Institute of Technology (KTH), 10044 Stockholm, \\ Sweden, E-mail jobo@jibs.hj.se
}

\begin{abstract}
A major characteristic of the economic development in European and North America during the past 10-15 years is a fast expansion of the producer-service sector. This paper considers the location dynamics of two categories of firms: contact-intensive producer-service suppliers and other firms, where the latter form the rest of the economy. Urban regions are decomposed into urban areas, and the latter into zones. In the theoretical framework firms have randomchoice preferences and respond in a non-linear way to time distances in their contact efforts. They make their location decisions in response to local, intra-regional and extra-regional access to market demand. This leads to a non-linear system that over time generates cumulative change processes of growth and decline. The econometric analysis makes use of information about time distances between zones in urban areas as well as between urban areas in the same agglomeration and between urban areas in different agglomerations. This information is employed in an econometric model that depicts for each urban area how the number of jobs in different sectors change in response to the access to customers' purchasing power in the entire set of urban areas. The estimation results show that the cumulative change processes feature non-linear behaviour.
\end{abstract}

Keyword: Agglomeration, business services, accessibility, non-linear JEL: R12, R12, L84 


\section{INTRODUCTION}

\subsection{Accessibility in Urban Agglomerations}

Economic growth take place in a spatially selective manner, and technological, social and lifestyle innovations are clustered in space, bringing about geographic concentrations of economic renewal. An urban region is a universe in its own right of such cocncentrations, where zones form urban areas, which in turn form urban regions

A major assumption is that firms benefit and become more productive from interaction with each other, due to interaction and communication externalities as phrased in Fujita and Thisse (2002). From another perspective, we may argue that individual firms can benefit from upstream and downstream externalities which bring about co-location advantages. Location properties of this kind can motivate the existence of urban regions (urban agglomerations), which consist of interlinked urban areas, which in turn can be decomposed into zones. For each of these levels, co-location advantages so that spatial concentrations can be observed at different levels of spatial resolution. This suggests that a system of urban agglomerations has a fractal property, with major centres, centres of urban areas as well as sub centre concentrations (Anas, Arnott and Small, 1998). An appropriate approach to analyse such a system is to identify accessibility measures to characterise an individual urban agglomeration.

Our focus is firms supplying business or producer services, and for these firms and their customer firms, the issue of interaction with direct face-to-face contacts seems to be a clear case where interaction externalities will prevail. In view of this we introduce a model in which firms supplying producer services make their location decisions as a response to the accessibility to customer demand (customer contacts) that each possible location offers. The accessibility measure introduced refer back to Weibull (1976). In this paper we follow Mattsson (1984) and Johansson and Klaesson (2008) when deriving the accessibility measure from a random-choice preference function of decision-making firms.

In view of the above, the present paper contributes to the modelling and the analysis of urban regions in five aspects. First, for the supply in each urban location, we apply a model which identifies many demand concentrations in a spatial landscape - consisting of urban areas, with zones, other urban areas belonging to the same region, and other urban regions. Second, the model specifies the time distance from a supply location to all demand concentrations. Third, for each supply location we derive the market access for producerservice suppliers from a random-choice decision model. Fourth, on the basis of each location's market access, we calculate the attractiveness of each possible supply location. Fifth, we specify and estimate the gradual change of the location of the producer-service supply as a function of every location's market access (or attractiveness). This is complemented by a similar specification of how other economic activities gradually develop across urban locations. These processes imply that the supply expand and shrink in different urban areas as well as in each urban region as a whole.

\subsection{Distance-Sensitive Transactions and Scale Economies}

The major focus in this paper is to understand urban locations as a place for communication externalities and interaction. We have chosen to selling activities of producer-service suppliers as major example of interaction between firms as buyers and firms as customers. The reason for this is (i) that producer-service suppliers have all firms as potential customers, and (ii) that the pertinent transactions can be classified as distance sensitive. 
Considering economic life in an urban region across all sectors, it can be depicted as a complex of transactions between buyers and sellers. Some of these transactions are distance sensitive in the sense that their probability (or frequency) declines fast as the time distance between supplier and customer increases, whereas other transactions are less sensitive.

The suppliers of producer services are assumed to supply distance-sensitive, differentiated product varieties under conditions of fixed costs. The demand for a suppliers output depends positively on the market access that obtains in the location of the firm, while the firm perceives its "own" negatively sloping demand schedule. This creates a market with properties resembling monopolistic competition, where the number of differentiated varieties will expand as the market access (accessibility to customer demand) expands (Fujita and Thisse, 2002; Johansson and Forslund, 2008). New varieties can be introduced only as the market access, because each supplier needs sufficiently large sales to cover costs. The described features sets the stage for the model of how market access stimulates the growth and decline of jobs in firms supplying producer services as well as for firms in the rest of the economy.

\subsection{Location Attractiveness and Growth}

An urban area with a large market access is likely to be large and to be part of a large urban agglomeration. When our model results show that the size of market access influences the growth of an urban area, this does not contradict previous model which tend to show that the size of urban regions affect their growth (e.g. Henderson, 1988; Glaeser, 1992, 2000; Forslund, 1998). The particular difference is that in the present model, the internal structure affects the change process of urban areas and their regions. Change in an urban area is modelled as a response to the structure of market access associated with location in that urban area, and these responses are different for different groups of economic activities.

The model considers change in three groups of activities: KP-firms supplying knowledgeintensive producer services, OP-firms supplying other producer services, and O-firms comprising the rest of the economy. Change is measured in terms of jobs in each sector and location. For each urban area, the model specifies how change processes (for each sector) are influenced by (i) local market access in the specific urban area, (ii) intra-regional market access, and (iii) extra-regional market access. Market access in a location reflects the discounted value of the economic activity (customer budget) in each of the three categories of markets (i) - (iii).

An outcome of the empirical estimations is that spatial pattern matters, and that producer services are associated with more distance-sensitive transactions than the urban economy as a whole. Regressions our carried through with a linear system and a quadratic system, demonstrating that the linear system leads to misinterpretations of the dynamics. We also show that the estimated model can be embedded in a sequence of periods to assess cumulative aspects of the change process when the market access vector develops as a consequence of gradually changing location of economic activities.

\subsection{Outline of the Paper}

Section 2 outlines the theoretical framework of the paper, presenting the map of the spatial organisation as viewed from each urban area, deriving the accessibility measures form a random-choice model, making the distinction between local, intra-regional and extra-regional, and introducing a model of supply behaviour of firms. Section 3 specifies equations that depict the change process for the three sectors, and presents 8 conjectured properties of the 
change processes, which are tested in the empiricalpart in Section 4. Concluding remarks are presented in Section 5, which also discusses further research opportunities.

\section{MEASURES OF MARKET ACCESS}

\subsection{Spatial organisation}

A basic feature of the spatial economy is a set of nodes where activities are concentrated. To detect nodes the space has to be observed via zones of predetermined size, and then some zones will contain dense concentrations and others not. Applying large zones to an urban agglomeration, few nodes will be identified, whereas small zonal areas will imply an increased number of nodes. In recent decades this phenomenon has been referred to as a fractal property of the urban landscape (Anas, Arnott and Small, 1998).

In view of this, this paper describe urban agglomerations as a set of nodes together with a corresponding set of links, which form a network, connecting the nodes. We shall consider three different layers of nodes. The medium layer consists of urban areas, which can be towns and cities, depending on the size. The lower layer consists of zones within an urban area, and the upper layer consists of functional urban regions, frequently referred to as just urban regions or urban agglomerations. In the limiting case, whenthe uran region is small, the region consists of only one urban area. In general, an urban region is a set of interconnected urban areas, between which the interaction is more intense than it is between urban areas belonging to different urban regions.

Each link connecting two urban areas, $r$ and $s$, is characterised by the time distance, $t_{r s}$, between the two areas. For an individual urban area we can also identify the average time distance, $t_{r r}$, between any pair of nodes within the area. Actually, the time distance between two urban areas is determined as the average between all zones in each of the two regions. The basic model is based on (i) the location of activities in each urban area and (ii) the time distance between each urban area.

The size of economic activities in an urban area $r$, is reflected by the total wage sum, $W_{r}$, in area $r$, generated by firms in the area. Economic activities are grouped into three sectors, where the KP-sector consists of firms supplying knowledge-intensive producer or business services, the OP-sector consists of firms supplying other producer services, and the O-sector consists of all other firms. For area $r$, we identify the number of jobs in the KP-firms, $L_{r}^{K P}$, in OP-firms, $L_{r}^{O P}$, and in other firms, $L_{r}^{O}$. The total number of jobs, is given by $L_{r}=L_{r}^{K P}+L_{r}^{O P}+L_{r}^{O}$.

Consider now a firm located in urban area $r$. Given this position our model considers three types of locations. The first is area $r$ itself, the second consists of areas $s \in R(r)$, which belong to the same urban region as $r$, and the third consists of all areas $k \in E(r)$, which belong to other urban regions. The focus of the analysis is the location decisions of suppliers of producer services and how these decisions depend on the location advantage of each area $r$. The advantage of a location in area $r$ will be measured by

(i) intra-urban market access, reflecting the accessibility to customer demand inside area $r$

(ii) intra-regional market access, reflecting the accessibility to customer demand in areas $s \in R(r)$, 
(iii) extra-regional market access, reflecting the accessibility to customer demand in areas $k \in E(r)$.

Once these measures are established, we assume that the supply of producer services in an urban area expands or contracts in response to the size of the accessible demand for the output from suppliers located in that area. In this way we can formulate equations for the location dynamics across urban areas.

In the sequel we assume that the wage sum $W_{s}$, reflects the size of economic activities in area $s$. In view of this, $W_{s}$ will also indicate the size of demand for producer services in area $s$. As outlined in Table 2.1, each supplier will be located in a particular zone in urban area $r$, but we consider only the average attributes across zones for each urban area (town or city). We may also note that the label functional urban region, which is used in Johansson and Klaesson (2008), is synonymous to our terms urban region and urban agglomeration, and that a metropolitan region is just a large urban region.

The travel times referred to in Table 2.1 are usually measured with reference to travel by car, although they should be thought of as travel time for the the most frequently used travel mode.

Table 2.1: Organisation of Urban Space

\begin{tabular}{|l|l|l|}
\hline \multicolumn{1}{|c|}{ Nodal layers } & \multicolumn{1}{c|}{ Time distances } & \multicolumn{1}{c|}{ Notation } \\
\hline Zone in an urban area & $\begin{array}{l}\text { Time distance inside a zone is } \\
\text { considered negligible }\end{array}$ & $\begin{array}{l}\text { Zones are the basis for } \\
\text { measuring time distances, but } \\
\text { are not identified in the models }\end{array}$ \\
\hline $\begin{array}{l}\text { Urban area } \\
\text { (municipality) }\end{array}$ & $\begin{array}{l}\text { An urban area consists of zones, } \\
\text { between which the average time } \\
\text { distance, } t_{r r}, \text { is about } 10 \\
\text { minutes. }\end{array}$ & $\begin{array}{l}\text { An urban area is denote by } r \text { and } \\
s .\end{array}$ \\
\hline $\begin{array}{l}\text { Urban region or urban } \\
\text { agglomeration (UR) }\end{array}$ & $\begin{array}{l}\text { The UR consists of urban areas, } \\
\text { between which the time } \\
\text { distances, } t_{r s}, \text { vary between } 15 \\
\text { and 50 minutes }\end{array}$ & $\begin{array}{l}R(r) \text { denotes all areas belonging } \\
\text { to the same urban region as } r, \\
\text { except } r \text { itself }\end{array}$ \\
\hline $\begin{array}{l}\text { Extra-regional } \\
\text { territory }\end{array}$ & $\begin{array}{l}\text { Urban areas belonging to other } \\
\text { regions. The average time } \\
\text { distance between urban areas in } \\
\text { two different regions exceeds } 60 \\
\text { minutes. }\end{array}$ & $\begin{array}{l}\text { E(r) denotes all areas belonging } \\
\text { to other regions than region } R \text { to } \\
\text { which } r \text { itself belongs }\end{array}$ \\
\hline
\end{tabular}

\subsection{Random Choice and Accessibility}

As described in the previous subsection, the market of suppliers of producer services is divided into three categories: intra-urban customers, intra-regional customers, and extraregional customers, which are located in other regions. We shall consider a measures of a supplier's access to customer demand, which is based on the assumption that suppliers make the effort to contact its customers. Although both suppliers and customers make contact efforts in many markets, we shall stick to a decision model, in which suppliers make the contact efforts. 
Following the modelling in Johansson, Klaesson and Olssson $(2002,2003)$, we assume that the supplier has a random-choice preference function $\tilde{V}_{r s}=V_{r s}+\varepsilon_{r s}$, which consists of a systematic part $V_{r s}$ and an extreme-value distributed random part, denoted by $\varepsilon_{r s}$. The systematic part is specified as follows:

$$
V_{r s}=\phi_{r s}-\lambda_{r s} t_{r s}
$$

where $t_{r s}$ is the time distance between $r$ and $s, \phi_{r s}$ reflects spatial preferences of customers in area $r$ with regard to area $s$, and $\lambda_{r s}$ is a time-sensitivity coefficient, where the parameters are specified in (2.2).

$$
\phi_{r s}=\left\{\begin{array}{l}
\hat{\phi}_{1} \text { as } r=s \\
\hat{\phi}_{2} \text { as } \mathrm{s} \in R(r) \\
\hat{\phi}_{3} \text { as } \mathrm{s} \in E(r)
\end{array} \quad \lambda_{r s}=\left\{\begin{array}{l}
\lambda_{1} \text { as } r=s \\
\lambda_{2} \text { as } \mathrm{s} \in R(r) \\
\lambda_{3} \text { as } \mathrm{s} \in E(r)
\end{array}\right.\right.
$$

Established results for a random-choice model of the type introduced here tell us that the probability, $P_{r s}$, of suppliers in area $r$ to contact customers in area s equals (Mattsson, 1984; Johansson and Klaesson, 2008):

$$
P_{r s}=W_{s} \exp \left\{V_{r s}\right\} / \sum_{s} W_{s} \exp \left\{V_{r s}\right\}
$$

where $W_{s}$ is the total wage sum in urban area $s$, which represents the total purchasing capacity in area $s$, which we assume is positively related to the demand for producer services. The variable $P_{r s}$ reflects the share of demand from customers in $s$. In view of this, the denominator of (2.3) can be used as an indicator of the demand potential of supply firms in urban area $r$. Because of this, the expression in the denominator is interpreted as area $r$ 's accessibility to customer demand, signified by $\sum_{s} W_{s} \exp \left\{V_{r s}\right\}=A_{r r} \exp \left\{\phi_{1}\right\}+A_{R(r)} \exp \left\{\phi_{2}\right\}$ $+A_{E(r)} \exp \left\{\phi_{3}\right\}$, in accordance with the specification in (2.3), where

$$
\begin{aligned}
& \hat{A}_{r r}=W_{r} \exp \left\{\phi_{1}-\lambda_{1} t_{r r}\right\}=A_{r r} \exp \left\{\phi_{1}\right\} \\
& \hat{A}_{R(r)}=\sum_{s \in R(r)} W_{s} \exp \left\{\phi_{2}-\lambda_{2} t_{r s}\right\}=A_{R(r)} \exp \left\{\phi_{2}\right\} \\
& \hat{A}_{E(r)}=\sum_{s \in E(r)} W_{s} \exp \left\{\phi_{3}-\lambda_{3} t_{r s}\right\}=A_{E(r)} \exp \left\{\phi_{3}\right\}
\end{aligned}
$$

In view of formula (2.4), we shall refer to the triplet $A_{r}=\left(A_{r r}, A_{R(r)}, A_{E(r)}\right)$ as the pure market access vector of location $r$. It can be translated to the overall market demand accessibility, $\hat{A}_{r}=\left(\hat{A}_{r r}, \hat{A}_{R(r)}, \hat{A}_{E(r)}\right)$ by means of the three coefficients, $\exp \left\{\phi_{1}\right\}, \exp \left\{\phi_{2}\right\}$ and $\exp \left\{\phi_{3}\right\}$.

The parameters in (2.4) have been estimated from a data set on trip making inside and between 288 Swedish urban areas by means of a multi-constrained trip-making model, with an objective function of entropy type (Johansson, Klaesson and Olsson, 2003). In this 
exercise, a series of empirical estimations were made for different types of trip makers. All these results reveal that the following pattern applies:

$$
\phi_{1}>\phi_{2}>\phi_{3} \text { and } \lambda_{2}>\lambda_{3}>\lambda_{1}
$$

which implies that the valuation of location advantages is governed by preferences that give priority to local proximity over intra-regional proximity, while the latter is preferred over extra-regional proximity. The other implication is that the time sensitivity is largest for medium-length intra-regional distances and smallest for very short local distances. We may refer to this as a property of non-linear time responses among decision makers. In particular, the constellation of the six parameters in (2.5) that preferences for contact making are highly non-linear, and these non-linearities are carried over to the accessibility measures.

\subsection{Local, Regional and Extra-Regional Market Access}

Consider the basic conditions of any firm, i.e., of firms in any of the three sector KP, OP and O. On the one hand the firm makes use of markets for buying inputs, and on the other it makes use of markets for selling its output. In both cases the firm's entire market is decomposed into local, regional and extra-regional market. Firms with distance-sensitive inputs benefit from having a large supply inside its own urban region, which for urban area $r$ would be reflected by large values of $A_{r r}$ and $A_{R(r)}$. These two market-access measures show the size of the opportunities of input suppliers to contact input-buying firms. Indirectly, $A_{r r}$ and $A_{R(r)}$ will also indicate the advantage for input buyers, the supply of inputs will be larger in an area $r$ where the market access variables are large.

The mirror case comprises suppliers of distance-sensitive producer services. In particular, these firms benefit from large values $A_{r r}$ and $A_{R(r)}$. These conclusions should imply that the location of producer services reflects the size of the intra-urban and intra-regional market access. In the present study we extend this idea, by assuming that the growth of producer services in a region is influenced by the market access vector $A_{r}=\left(A_{r r}, A_{R(r)}, A_{E(r)}\right)$, which corresponds to the idea that firms adjust their location and the size of their supply from a given location in response to the $A_{r}$-vector, and while doing that they also influence the $A_{r}$ vector in a cumulative way.

It is evident that the $A_{r}$-vector is especially important for suppliers of producer services, since service supply is considered distance sensitive in a pronounced way. However, market access is relevant for all suppliers (firms). For firms with moderate and low distance sensitivity, the extra-regional market access may be relatively more important than for suppliers of producer services.

\subsection{Agglomeration Economies and Market Access}

Having reached this far, let us ask: why does the size of market access, as expressed by the $A_{r}$-vector matter? In view of results from models concerning the economics of agglomeration (Fujita and Thisse, 2002), the size of the accessible market determines whether a certain location is feasible for a firm that supplies a differentiated, distance-sensitive product under conditions of (i) monopolistic competition, and (ii) fixed costs. A simple model of this type is 
presented in Johansson and Forslund (2008) for the case where each firm i supplies a differentiated variety $i$, and Andersson and Johansson (2008) for the case where a firm may supply several varieties.

Table 2.2 presents the simple version of the model, for which every firm supplies its own differentiated product variety. In the table $x_{i r}$ denotes demand for product variety $i$ when $r$ is the supply point, $G_{i}\left(A_{r}\right)$ is an increasing function of the market-access vector $A_{r}$ for suppliers in $r, p_{i}$ denotes price, $v_{i}$ the variable cost, $F_{i}$ the fixed cost, $\delta_{i}$ a slowly changing coefficient, and $\theta$ is the price elasticity.

This means that formula (i) and (ii) in Table 2.2 correspond to a standard description of monopolistic competition in the New Economic Geography tradition. In formula (iii) we have added the variable $\rho_{r}=\rho\left(A_{r}\right)$, which denotes the land rent per unit output, where $\rho$ is increasing in $A_{r}$. The location criterion is given by formula (iii), which shows how the average cost shrinks as the realised sales increase, recognising that the firm's sales depend on its market access, given optimal price setting.

Table 2.2: Monopolistic competition, market access and feasible locations

\begin{tabular}{|l|l|}
\hline \multicolumn{1}{|c|}{ Formula } & \multicolumn{1}{c|}{ Interpretation } \\
\hline (i) $x_{i r}=\delta_{i} G_{i}\left(A_{r}\right) p_{i}^{-\theta}$ & $\begin{array}{l}\text { Demand function of firms, where } G_{i}\left(A_{r}\right) \text { depicts firm } i \text { 's } \\
\text { access to the purchasing power when located in area } r \text {, and } \\
\text { where } \theta_{i} \text { is the price elasticity }\end{array}$ \\
\hline (ii) $p_{i}=\theta /(\theta-1) v_{i}$ & $\begin{array}{l}\text { The optimal price selected by firm } i \text { in an environment of } \\
\text { monopolistic competition, where } v_{i} \text { represents marginal cost }\end{array}$ \\
\hline (iii) $p_{i} \geq v_{i}+F_{i} / x_{i r}+\rho_{r}$ & $\begin{array}{l}\text { This is the condition for a feasible location in } r \text {, which requires } \\
\text { that } x_{i r} \text { is large enough to make } F_{i} / x_{i r} \text { small enough, where } F_{i} \\
\text { represents fixed costs. }\end{array}$ \\
\hline
\end{tabular}

According to (ii) $p_{i}>v_{i}$. Thus, condition (iii) will be satisfied as soon as $x_{i r}$ becomes large enough to make $F_{i} / x_{i r}$ small enough. Moreover, for a given set of supplying firms, $x_{i r}$ grows as $A_{r}$ grows. At the same time $\delta_{i}$ shrinks as more firms enter the set of differentiated suppliers. As a consequence, the size of the market access vector stimulates firms to locate in $r$, while a growth in $\rho_{r}=\rho\left(A_{r}\right)$, as $A_{r}$ increases, counteracts the demand-expansion effect of a growing market access. As the size of the market access of an urban area increases, this implies that the demand for more varieties increases, and hence more suppliers can establish themselves in the urban area. The principle temporal mechanism in this stylised model is then that (i) the size of the $A_{r}$-vector stimulates the entry of a greater supply, and (ii) the expanded supply adds to the size of the $A_{r}$-vector.

There are two growth dampening forces. One works via increased land rents, the second works via increased time distances, caused by an augmented $A_{r}$-vector that may increase density and time delays in contact efforts. The very formulation of an accessibility patterns recognises this phenomenon. However, in this model version, alterations of time distances are not considered. 


\section{LOCATION DYNAMICS OF BUSINESS SERVICES}

\subsection{Service Suppliers and Access to Customers}

Given the specifications of market conditions in Section 2, we want to study in a discrete-time setting how the size of producer-service supply changes between time $t$ and $t+\tau$. This change can be observed as an increase in (i) the value of the supply of services, (ii) the number of firms supplying producer services, and (iii) the number of jobs in firms supplying producer services. We will in the subsequent analysis study the process as a change in number of jobs.

Referring to urban area $r$, we define $L_{r}^{j}$ as the number of jobs in sector $j$, where $j=\mathrm{KP}$ refers to knowledge-intensive producer services, $j=$ OP refers to other producer services. In subsection 3.2 we also introduce $j=\mathrm{O}$ referring to all other sectors, representing the rest of the economy.

The change in $L_{r}^{j}$ is specified as $\Delta L_{r}^{j}=L_{r}^{j}(t+\tau)-L_{r}^{j}(t)$, and it is assumed to be a function of the $A_{r}$-vector, where each component has its own influence on the change process. Why should $L_{r}^{j}$ grow? One way of thinking is to assume that the market access, as given by $A_{r}$ allows for a supply size, $L_{r}^{j *}$, that exceeds $L_{r}^{j}(t)$, and hence the change can be characterised as an adjustment process. Given that adjustments cannot be considered instantaneous, the time interval $\tau$ should be long enough to allow responses to take place. At the same time, the time interval should not be too long, since $\Delta L_{r}^{j}$ adds to the total economic activity in area $r$, and because of this the accessibility factor $A_{r r}$ will increase, which in a cumulative way brings about further growth adjustments. Thus, if the time interval $\tau$ is too long it will not accureately reflect change between $t$ and $t+\tau$ as a response to the access pattern at time $t$.

When studying the change process of each sector's supply size, we will consider two specifications, on for a linear system and one for a quadratic system. The linear-system specification can be written as follows:

$$
\left.\Delta L_{r}^{j}=\alpha_{0}+\alpha_{1} A_{r}+\alpha_{2} A_{R(r)}+\alpha_{3} A_{E(r)}\right)
$$

showing how local, intra-regional and extra-regional market access stimulates the change. This may be seen as a direct application of formula (2.4), given that

$$
\begin{aligned}
& \hat{A}_{r r}=k_{1} A_{r r} \exp \left\{\phi_{1}\right\}=\alpha_{1} A_{r r} \\
& \hat{A}_{R(r)}=k_{2} A_{R(r)} \exp \left\{\phi_{2}\right\}=\alpha_{2} A_{R(r)} \\
& \hat{A}_{E(r)}=k_{3} A_{E(r)} \exp \left\{\phi_{3}\right\}=\alpha_{3} A_{E(r)}
\end{aligned}
$$

where $k_{1}, k_{2}$ and $k_{3}$ are three response parameters. Interpreting formula (3.1), we can see that if there is a lowest level for the compound market access, below which no supply is possible from urban area $r$, then this means that $\alpha_{0}<0$. A second observation is that demand in locations $r$ and $s \in R(r)$ represent the "home-market" areas for suppliers in $r$. Thus, $\alpha_{1}>0, \alpha_{2}>0$, and $\alpha_{3}<0$ will express that large extra-regional market access provides incentives to locate outside the region to which $r$ belongs. 
The change process can come to rest only if at least one of the $\alpha$-coefficients is negative. Such a dynamic equilibrium is obviously never stable, but we may contemplate grouping $A_{r}$ vectors into domains in which $L_{r}^{j}$ invariantly remains positive or negative. As the paper aims at showing that equation (3.1) is not sufficient for revealing the basic properties of the agglomeration change processes, we turn to an a equation system with quadratic form in (3.3):

$$
\Delta L_{r}^{j}=\alpha_{0}+\alpha_{1} A_{r}+\alpha_{2} A_{R(r)}+\alpha_{3} A_{E(r)}+\beta_{1}\left(A_{r}\right)^{2}+\beta_{2}\left(A_{R(r)}\right)^{2}+\beta_{3}\left(A_{E(r)}\right)^{2}
$$

This formulation cannot ascertain the direct relation between $\phi$-parameters and $\alpha$ parameters. The reason for this is that (3.3) assumes that the response mechanism are not linear in the market-access vector. In this context we especially observe that the constellation of parameters will have to reflect the net effect of (i) expanding market access and (ii) increased land rents for each urban area.

When leaving the linear specification, we attempt to model response mechanisms which can reflect our basic market-access assumptions. These assumptions may be specified as follows: For contact-intensive and distance-sensitive producer services we should expect the following parameter values:

Property 1: $\alpha_{0}<0$ to reflect that there is threshold level of the market access vector, below which an urban area will not experience growth in sectors where $j=\mathrm{K}$ and $j=\mathrm{OP}$.

Property 2: $\alpha_{1}>0$ reflects that the local market access generates a positive stimulus to expansion of producer services. If, in addition, $\beta_{1}>0$ the growth response is accelerating in the local market access.

Property 3: A pair $\left[\alpha_{2}<0, \beta_{2}>0\right]$ reflects that there is a positive growth stimulus as $A_{R(r)}$ is sufficiently large.

Property 4: $\alpha_{3}<0$ reflects a negative growth response to "intervening opportunities" in neighbouring urban agglomerations. If, in addition $\beta_{3}>0$, this reflects that growth stimulus may obtain if sufficiently large extra-regional market access is present.

Properties 1 and 2 are self-explanatory. For distance-sensitive producer services, we may expect that the property $\left[\alpha_{1}>0, \beta_{1}>0\right]$ applies. Property 3 is referred to as a J-shaped response mechanism: for low values of $A_{R(r)}$ the impact is negative and turns positive as $\beta_{2}\left(A_{R(r)}\right)^{2}>-\alpha_{2} A_{R(r)}$, and the derivative $\partial \Delta L_{r}^{j} / \partial A_{R(r)}>0$ as $2 \beta_{2} A_{R(r)}>-\alpha_{2}$. The J-shaped response is illustrated in Figure 3.1.

\subsection{Comprehensive Job Dynamics}

The outline in the preceding subsection is focussed on suppliers of producer services and their change process. That will only provide a partial description of job dynamics for each urban area. However, when we extend the time horizon the cumulative growth phenomenon will affect all the market access variables, and to describe how they change we need a 
comprehensive system of job dynamics. Thus, besides firms in the sector KP and OP, we also must control for the change, $\Delta L_{r}^{O}$, of jobs in the rest of the economy, i.e., the O-sector.

In the model formulation in this paper, we have chosen to depict the change $\Delta L_{t}^{O}$ during the time interval $(\mathrm{t}, \mathrm{t}+\tau)$ in the way specified by equation (3.1) and (3.3), where (3.1) remains a linear system, and we intend to use our econometric analysis to illustrate that also for $\mathrm{O}$-sector firms the location dynamics are better represented by the non-linear system as given by (3.3).

A major reason for treating the $\mathrm{O}$-sector firms separately is that on average these firms are less contact intensive and engaged in less distance-sensitive interactions. Important parts of the O-sector are manufacturing firma and household service suppliers, and many of the firms in the O-sector have a major part of their customers outside their own urban agglomeration. With regard to the quadratic specification in (3.3) these characteristics imply the following expectations about parameter values for the $\mathrm{O}$-sector:

Property 5: $\alpha_{0}<0$ for the same reasons as those given in Property 1.

Property 6: $\alpha_{1}>1$, expressing that local market access is important also for $\mathrm{O}$-firms. If the local market access is a weaker stimulus for the $\mathrm{O}$-sector firms, this would imply that we should observe the pair $\left[\alpha_{1}>0, \beta_{1}<0\right]$, which means that the growth response is positive for urban areas of moderate size, while it turns negative as $A_{r r}$ becomes large.

Property 7: The pair $\left[\alpha_{2}>0, \beta_{2}<0\right]$ reflects that $\mathrm{O}$-firms are stimulated by a large intraregional market access, but beyond a certain size, the stimulus to growth disappears.

Property 8: The O-sector firms benefit from extra-regional market access. If this phenomenon is vary pronounced, we should observe $\left[\alpha_{3}>0, \beta_{3}>0\right]$

Properties 1-8 express what we expect from theoretical arguments in Section 2, and all these hypotheses will be tested in the empirical estimations of Section 4. We may observe that we assume more similarities than differences between O-sector firms and producer-service suppliers. One should also add that the differences between the three types of sectors are reflected both by sign and size of parameters.

Observe also that Property 6 and 7 differ from Property 3 and 4. The former are assumed to be concaved-shaped, whereas the latter are assumed to be J-shaped (Figure 3.1). 
$\Delta L_{r}^{j}$

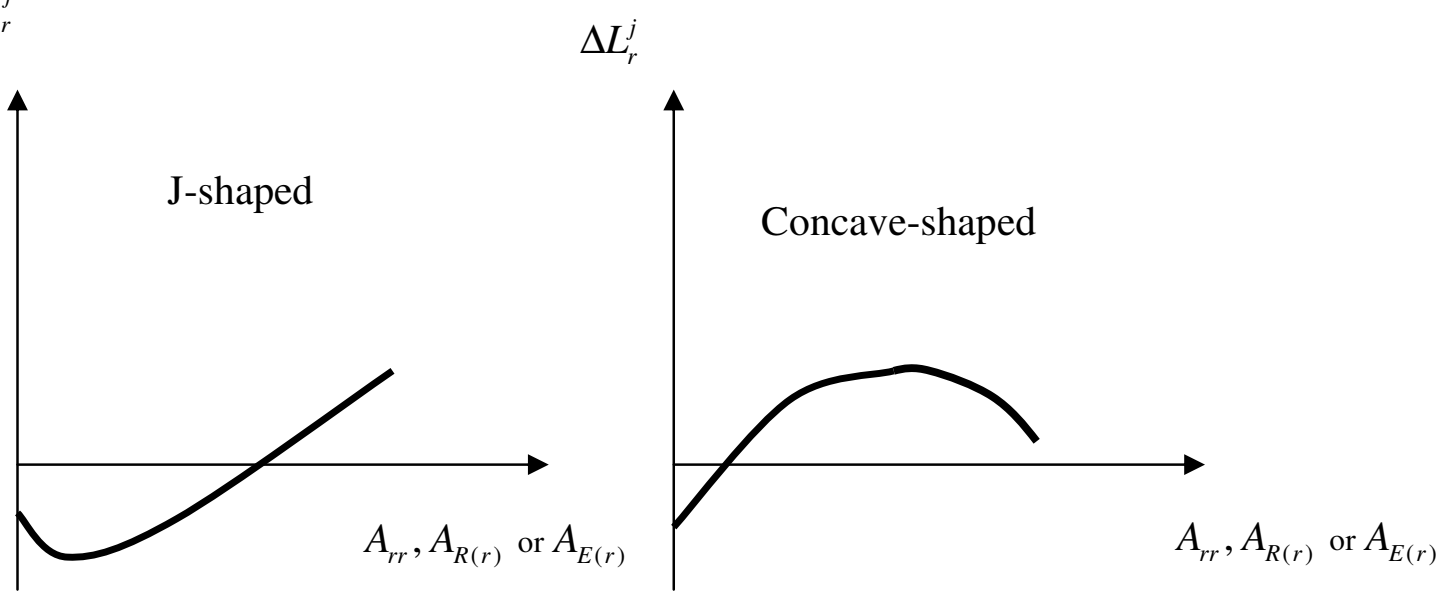

Figure 3.1: Response to market access components

\subsection{Feed Back Dynamics and Agglomeration Growth}

The model of the change process of the spatial economy as specified in (3.3) describes change during a time interval $\tau$. However, it is possible to specify a sequence of $\tau$-periods, with observations of each urban region's market access at dates $\mathrm{t}, t+\tau, \mathrm{t}+2 \tau$, etc. To trace the change process over a sequence of periods, we need information about the wage level in each of the three sectors.

The estimated model of the change process generates vector of job changes, [ $\Delta L^{K P}(t+\tau)$, $\left.\Delta L^{O P}(t+\tau), \Delta L^{O}(t+\tau)\right]$ between $\mathrm{t}$ and $(t+\tau)$. This results in new values jobs, $\left[L^{K P}(t+\tau)\right.$, $\left.L^{O P}(t+\tau), L^{O}(t+\tau)\right]$ at time t, which together with the wage levels $\left[w_{r}^{K P}, w_{r}^{O P}, w_{r}^{O}\right]$ form the basis to calculate a new wages sum for each urban area $r$ at time $(t+\tau)$, as given by

$$
W_{r}(t+\tau)=\sum_{j} w_{r}^{j} L_{r}^{j}(t+\tau)
$$

where the $w_{r}^{j}$-values may be the wage levels at the starting date or wage levels given from a regression where $w_{r}^{j}(t)$ is a function of the $A_{r}(t)$ vector at each time $t$.

In the data set for the ampirical application of the model there is information about the wage level of each sector $j$ in each urban area $r$ at time $t$ and $t+\tau$, denoted by $w_{r}^{j}(t)$ and $w_{r}^{j}(t+\tau)$. The ambition of the following exercise is to detect the cumulative features of an estimated change process. Thus, for time periods ahead of $t+\tau$ it is meaningful to freeze the wage levels in each urban region to the levels at time $t+\tau$. The forward dynamics will then reveal the "isolated" effect of the changing spatial organisation - for a fixed-wage setting. A second alternative would be to estimate $w_{r}^{j}(t+\tau)$ as a function of the $A_{r}(t)$-vector or the $A_{r}(t+\tau)$-vector. In any case, it is possible to construct a sequence $w_{r}^{j}(t), w_{r}^{j}(t+\tau)$, 
$w_{r}^{j}(t+2 \tau)$, etc. In this way the dynamics of the spatial system can be examined as described in Figure 3.2, where the calculation of new wage-sum values in each urban area is essential for tracing the development of the market-access variables for each area. The second process $A_{r}(t)$ to keep track of is the step-wise change of job location. These two categories of variables determine the change in the market access vectors, given that time distances remain invariant.

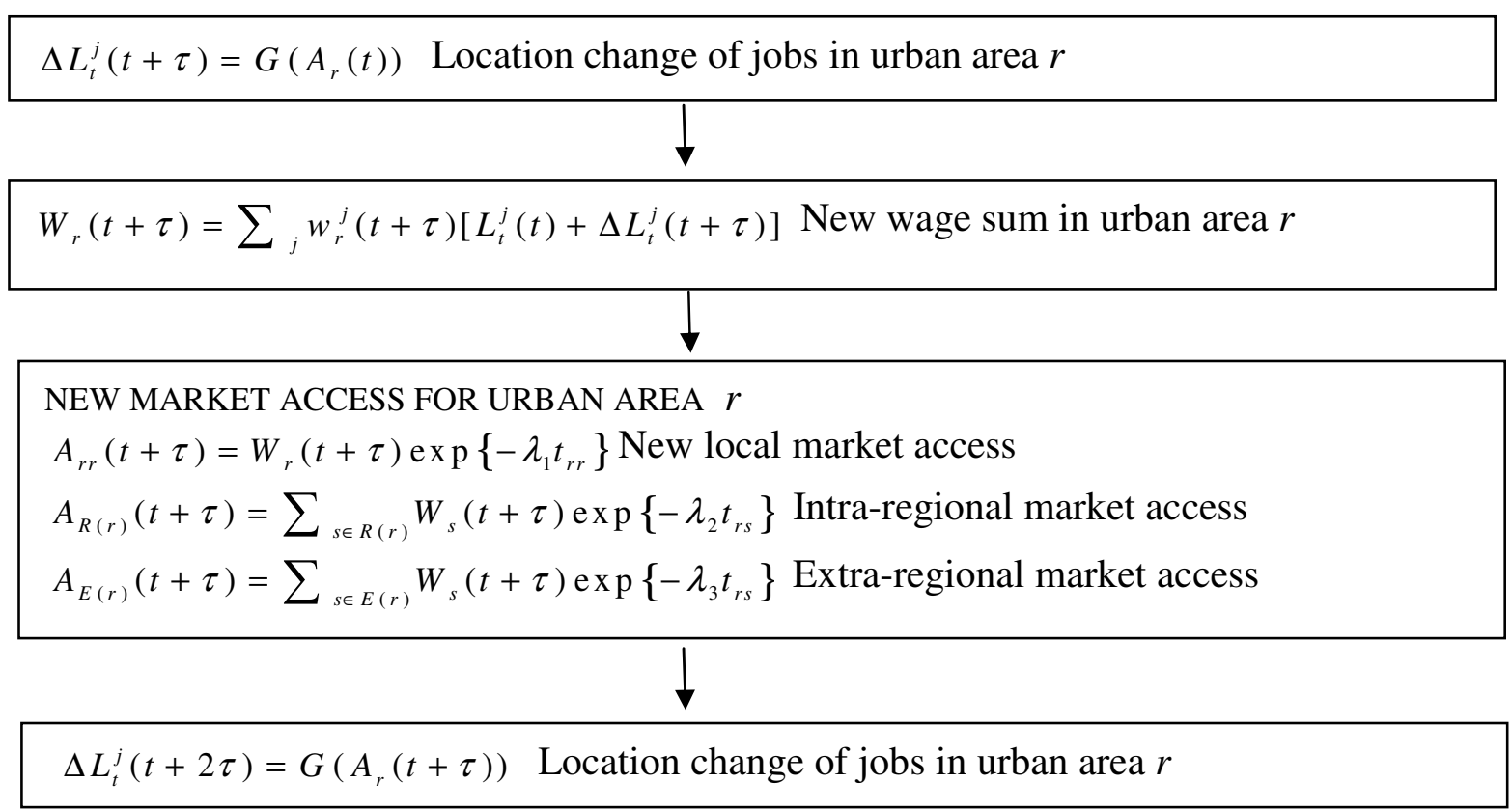

Figure 3.2: Sequential development of job location, market access and wage sum across urban areas

\section{ESTIMATION OF THE CHANGE PROCESS IN SWEDISH URBAN AREAS}

\subsection{Background Statistics}

All sectors of an urban economy consists of firms which are customers to suppliers of producer services. The purchasing power of those customers is reflected by the proxy variable by the wage sum in each urban region. As a background to the econometric analysis, Table 4.1 presents the wage sum in the three metropolitan regions, in the group of medium-sized regions, and in the group small regions.

Table 4.1: The wage sum (W) in Swedish urban regions 1999 and 2006

\begin{tabular}{|l|c|c|c|}
\hline Urban region & $\begin{array}{c}1999 \\
\text { (billion SEK) }\end{array}$ & $\begin{array}{c}2006 \\
\text { (billion SEK) }\end{array}$ & $\begin{array}{c}\text { Percentage } \\
\text { growth }\end{array}$ \\
\hline Stockholm & 243 & 300 & 23 \\
\hline Göteborg & 90 & 117 & 30 \\
\hline Malmö & 80 & 101 & 26 \\
\hline
\end{tabular}




\begin{tabular}{|l|c|c|c|}
\hline Medium-sized regions & 259 & 310 & 20 \\
\hline Small regions & 99 & 116 & 17 \\
\hline
\end{tabular}

Source: Statistics Sweden

The three measures of market access are such that their values increase with the size of an urban region. In particular, the $A_{R(r)}$-measure varies strongly with total employment of a region. Moreover, the $A_{r r}$-measure of the largest urban area in a region increases in concordance with the absolute size of the region. As a consequence, we should expect exactly the pattern which is presented in Table 4.2. There we can see that the share of KP-service jobs attains the highest value in the largest urban region (Stockholm), the second highest in the second largets region (Göteborg), and continues to fall with the size of each urban region. The smallest share (in small regions) is less than one third of that in the Stockholm region for the year 2006. This pattern holds both for KP-services and OP-services.

Table 4.2: Share of producer services in urban regions 1999 and 2006

\begin{tabular}{|l|c|c|c|c|}
\hline Urban region & $\begin{array}{c}\text { KP-services } \\
1999\end{array}$ & $\begin{array}{c}\text { KP-services } \\
2006\end{array}$ & $\begin{array}{c}\text { OP-services } \\
1999\end{array}$ & $\begin{array}{c}\text { OP-services } \\
2006\end{array}$ \\
\hline Stockholm & 8.56 & 11.07 & 27.51 & 30.46 \\
\hline Göteborg & 6.82 & 8.45 & 21.56 & 24.03 \\
\hline Malmö & 5.46 & 8.02 & 19.15 & 22.77 \\
\hline Medium-sized regions & 3.76 & 4.32 & 14.97 & 16.55 \\
\hline Small regions & 2.42 & 2.73 & 12.19 & 12.96 \\
\hline
\end{tabular}

Remark: Share of persons employed in producer service sectors to total employment. Statistics Sweden

Table 4.2 tells us that the share of producer services is growing between 1999 and 2006. The table also shows that the growth of KP-services is much faster in the three metropolitan regions than in either medium-sized or small regions. This is further illuminated in Table 4.3. The growth rate for OP-services is more evenly spread across regions that are medium-sized or larger. At the same time the growth rate is about half as high for the small regions. Other sectors of the economy grow very little and the growth rate is negative in Stockholm and Norrköping \& Linköping. The table also indicates that total urban growth is higher for those regions which have a high growth rate in producer services.

Table 4.3: Growth of employment in sectors 1999 - 2006 in percent

\begin{tabular}{|l|c|c|c|c|}
\hline Urban region & KP-services & OP-services & $\begin{array}{c}\text { Other } \\
\text { sectors }\end{array}$ & All sectors \\
\hline Stockholm & 39.89 & 19.71 & -1.14 & 8.11 \\
\hline Göteborg & 41.15 & 27.13 & 7.52 & 14.04 \\
\hline Malmö & 65.81 & 34.16 & 3.58 & 12.83 \\
\hline Medium-sized regions & 24.65 & 19.94 & 5.68 & 8.53 \\
\hline Small regions & 17.75 & 11.00 & 3.12 & 4.44 \\
\hline
\end{tabular}

Remark: Share of persons employed in producer service sector to total employment. Statistics Sweden 


\subsection{Assessment of the Econometric Estimation with the Linear System}

Formula (3.1) specifies a linear model of sector growth, where the change of jobs in a sector has the form $\Delta L_{r}^{j}=\alpha_{0}+\alpha_{1} A_{r}+\alpha_{2} A_{R(r)}+\alpha_{3} A_{E(r)}$, which means that the sector change is a linear function of intra-urban, intra-regional and extra-regional market access. In line with our previous theory discussion, Table 4.1 presents results, where producer-service jobs respond positively and with significant parameters to the size of intra-urban or local market access, whereas the growth coefficients for intra-regional market access is positive but with less clear significance.

Table 4.1: Linear growth model. Sequence of three 5-year periods, 1999-2006

\begin{tabular}{|c|c|c|c|c|c|}
\hline & & & $\Delta K P$ & $\triangle O P$ & $\Delta O$ \\
\hline Intercept & & $\left(\alpha_{0}\right)$ & $\begin{array}{l}-160.31 \\
(5.49)^{* * *}\end{array}$ & $\begin{array}{l}-187.47 \\
(5.39)^{* * *}\end{array}$ & $\begin{array}{l}493.45 \\
(3.23)^{* * *}\end{array}$ \\
\hline Intra-urban & $A_{r r}$ & $\left(\alpha_{1}\right)$ & $\begin{array}{l}673.63 \\
(63.72)^{* * *}\end{array}$ & $\begin{array}{l}867.92 \\
(68.97)^{* * * *}\end{array}$ & $\begin{array}{l}-1011.84 \\
(18.32)^{* * * *}\end{array}$ \\
\hline Intra-regional & $A_{R(r)}$ & $\left(\alpha_{2}\right)$ & $\begin{array}{l}3.23 \\
(0.76)\end{array}$ & $\begin{array}{l}13.18 \\
(2.60)^{* * * *}\end{array}$ & $\begin{array}{c}18.830 \\
(0.85)\end{array}$ \\
\hline Extra-regional & $A_{E(r)}$ & $\left(\alpha_{3}\right)$ & $\begin{array}{c}-179.68 \\
(3.20)^{* * *}\end{array}$ & $\begin{array}{c}-115.00 \\
(1.72)^{*}\end{array}$ & $\begin{array}{c}427.74 \\
(1.46)\end{array}$ \\
\hline $\begin{array}{l}\text { R-sq. } \\
\text { (overall) }\end{array}$ & & & 0.90 & 0.92 & 0.52 \\
\hline
\end{tabular}

Between effects estimation. Absolute value of $\mathrm{t}$ statistics in parentheses. * significant at $10 \%$; ** significant at $5 \%$; *** significant at $1 \%$

The major result from Table 4.1 is that with the linear specification, the sign as well as size of each parameter are similar for the KP-sector and O-sector. For these two categories of producer-service supply, we can conclude the following with regard the growth of the supply:

(i) There is a threshold level for growth, indicated by $\alpha_{0}<0$.

(ii) The size of the local market access parameter $\alpha_{1}>0$ shows that the local market access has a large and significant impact on the growth of producer-service supply.

(iii) The response parameter $\alpha_{2}>0$, representing the growth impact of intra-regional market access, is positiuve, small, and not significant for KP-services.

(iv) The For KP-services, the response to extra-regional market access is significantly negative, expressed by $\alpha_{3}<0$.

In previous studies we have interpreted $\alpha_{3}<0$ as a "Christaller effect" showing that when a given urban area is close to one or several large extra-regional markets, then this could imply that jobs will rather grow in these extra-regional locations (Johansson and Klaessson, 2007). This may be labelled as an inter-regional competition effect. However, when we in the next subsection employ a quadratic specification, the understanding of the extra-regional market influences is improved considerably.

Table 4.1 also shows that the group other sectors has a different change pattern, such that there is a negative response to intra-urban (local) market access, while the response to intraregional and extra regional market access is not significant. A particular feature of the Osector in Table 4.1 is that threshold parameter, $\alpha_{0}$, which ten seems to imply that there is no size effect for the rest of the economy. However, as a quadratic specification is introduced, the econometrics will detect a threshold effect also ofr the O-sector. 


\subsection{Assessment of the Econometric Estimation with the Quadratic System}

The overall impression from Table 4.2 is that the quadratic specification generates parameter values that reflect a higher degree of similarity between the change process of the three economic sectors. This also allows for conjectures of a general response patter, where sectors differ in the strength of the response rather than in the sign of parameter values. Moreover, the nature of the differences between the sectors are better understood with the quadratic specification

Table 4.2: Non-linear growth model. Sequence of three 5-year periods, 1999-2006

\begin{tabular}{l|ccccc}
\hline Intercept & & & $\begin{array}{c}\Delta K P \\
-34.394\end{array}$ & $\begin{array}{c}\Delta O P \\
-111.070\end{array}$ & $\begin{array}{c}-180.537 \\
(1.24)\end{array}$ \\
\hline Intra-urban & & $\left(\alpha_{0}\right)$ & $(2.55)^{* *}$ & $(1.69)^{*}$ \\
\hline Intra-regional & \multirow{2}{*}{$A_{r r}$} & $\left(\alpha_{1}\right)$ & $\begin{array}{c}392.583 \\
(19.62)^{* * *}\end{array}$ & $\begin{array}{c}802.878 \\
(25.49)^{* * *}\end{array}$ & $\begin{array}{c}825.279 \\
(10.68)^{* * *}\end{array}$ \\
& $A_{R(r)}$ & $\left(\alpha_{2}\right)$ & -9.070 & -32.396 & 85.074 \\
& & & $(1.00)$ & $(2.27)^{* *}$ & $(2.43)^{* *}$ \\
\hline Extra-regional & \multirow{2}{*}{$A_{E(r)}$} & $\left(\alpha_{3}\right)$ & -216.322 & -154.495 & -5.847 \\
& & & $(2.63)^{* * *}$ & $(1.19)$ & $(0.02)$ \\
\hline Intra-urban & $\left(A_{r r}\right)^{2}$ & $\left(\beta_{1}\right)$ & 15.325 & 3.791 & -100.114 \\
& & & $(15.31)^{* * *}$ & $(2.41)^{* *}$ & $(25.91)^{* * *}$ \\
\hline Intra-regional & $\left(A_{R(r)}\right)^{2}$ & $\left(\beta_{2}\right)$ & 1.132 & 2.739 & -6.727 \\
& & & $(2.29)^{* *}$ & $(3.52)^{* * *}$ & $(3.53)^{* * *}$ \\
\hline Extra-regional & $\left(A_{E(r)}\right)^{2}$ & $\left(\beta_{3}\right)$ & 56.262 & 24.625 & 60.239 \\
& & & $(1.24)$ & $(0.35)$ & $(0.35)$ \\
R-sq. (overall) & & & 0.93 & 0.92 & 0.82 \\
\hline
\end{tabular}

Between effects estimation. Absolute value of $\mathrm{t}$ statistics in parentheses

* significant at $10 \%$; ** significant at $5 \%$; *** significant at $1 \%$

It is meaningful to discuss the parameter values in groups, referring to (i) threshold level, (ii) local market access, (iii) intra-regional market access, and (iv) extra-regional market access.

Threshold level: All three sectors are similar in having $\alpha_{0}<0$, although the the size of the parameter is small for the KP-sector. Thus there is a threshold level for growth.

Local market access: The growth response to the size of local market access has the same structure for the KP-sector and OP-sector, reflected by $\left[\alpha_{1}>0, \beta_{1}>0\right]$, which corresponds to an exponentially increasing reponse to the size of $A_{r r}$. For the O-sector, the response is given by the pair $\left[\alpha_{1}>0, \beta_{1}<0\right]$, showing that for $\mathrm{O}$-activities (as a group), the response remains positive as long as the local market access is not too large, and it turns negative as $A_{r r}$ exceeds a given size level, i.e., when $A_{r r}>-\alpha_{1} / \beta_{1}$. This could indicate that O-sector firms do not benefit enough from a large local market to compensate for the higher land rents that are associated with a large value of $A_{r r}$. 
Intra-regional market access: For the producer-service sectors, KP and OP, we observe $\left[\alpha_{2}<0, \beta_{2}>0\right]$, signifying a positive growth response that is initiated when $A_{R(r)}$ passes the threshold level $A_{R(r)}>-\alpha_{2} / \beta_{2}$. Thus, a large intra-regional market stimulates growth of producer-service supply. On the other hand, as the intra-regional market access becomes larger, the stimulus to growth of the O-sector is weakened, because in this case we have $\left[\alpha_{2}>0, \beta_{2}<0\right]$

Extra-regional market access: The overall results is that across the three sectors, there is only one parameter estimate that is significantly different from zero. The significant parameter is $\alpha_{3}<0$ for the KP-sector, indicating competition between regions with regard to knowledge-intensive producer services.

\section{CONCLUDING REMARKS}

A major result from the empirical exercise in this paper is that the macro patterns of firms' location choices in a temporal setting, evolve in response to the inner structure of each urban area, to each urban area's intra-regional accessibility to the other urban areas of the pertinent region, and to each urban area's accessibility to other regions.

The estimation results for the producer-service change process follow to a large extent the theoretical motivations in Section 3.1, which is illustrated in Table 5.1. However, one empirical result is a clear surprise. The hypothesis that the extra-regional influence has a negative influence on the growth, $\alpha_{3}<0$, has to be rejected for the OP-sector, for which the parameters $\alpha_{3}$ and $\beta_{3}$ are not significant. For the KP-sector $\alpha_{3}$ is negative but very small, while $\beta_{3}$ is not significantly different from zero. Thus, extra-regional features come out as not important.

A second observation is that for the local market access, the parameters $\alpha_{1}$ and $\beta_{1}$ are large and significant for the producer-service sectors. With regard intra-regional market access, it is the $\beta_{2}$-parameter that governs the growth process in the smaller urban areas in a region get growth, because for these regions the $A_{R(r)}$-value dominates.

Table 5.1: Suggested properties for the change process of producer-service supply

\begin{tabular}{|ll|ll|}
\hline Estimation result & & Suggested property & \\
\hline$\alpha_{0}<0$ & Threshold & $\alpha_{0}<0$ & Property 1 \\
\hline$\left[\alpha_{1}>0, \beta_{1}>0\right]$ & Exponential & {$\left[\alpha_{1}>0, \beta_{1}>0\right]$} & Property 2 \\
\hline$\left[\alpha_{2}<0, \beta_{2}>0\right]$ & J-shaped & {$\left[\alpha_{2}<0, \beta_{2}>0\right]$} & Property 3 \\
\hline$\left[\alpha_{3}<0, \beta_{3}>0\right]$ & J-shaped & $\alpha_{3}<0$ & Property 4 \\
\hline & & & \\
\hline
\end{tabular}

Table 5.2 compares the estimation results for the O-sector (rest of the economy) with Property 5-8. A major finding is that as soon as the $A_{R(r)}$ has a comparatively large value, then the change process is governed by $\beta_{1}$ and $\beta_{2}$, whereas the "linear parameters", $\alpha_{1}$ and $\alpha_{2}$, remain influential only for the largest urban areas, in particular the cities of the three 
metropolitan regions. Moreover, for the O-sector the influence from extra-regional market access is not significantly different from zero.

For the very large urban areas, the estimated coefficients $\alpha_{1}$ and $\beta_{1}$ imply that the producerservice supply develops in an accelerating way, whereas the O-sector will gradually decrease as the factor $\left(A_{r r}\right)^{2}$ gets large enough compared to $A_{r r}$.

Table 5.2: Suggested properties for the change process of the rest of the economy (O-sector)

\begin{tabular}{|ll|ll|}
\hline Estimation result & & Suggested property & \\
\hline$\alpha_{0}<0$ & Threshold & $\alpha_{0}<0$ & Property 5 \\
\hline$\left[\alpha_{1}>0, \beta_{1}<0\right]$ & Concave & {$\left[\alpha_{1}>0, \beta_{1}<0\right]$} & Property 6 \\
\hline$\left[\alpha_{2}>0, \beta_{2}<0\right]$ & Concave & {$\left[\alpha_{2}>0, \beta_{2}<0\right]$} & Property 7 \\
\hline$\left[\alpha_{3}<0, \beta_{3}>0\right]$ & J-shaped & $\alpha_{3}>0$ & Property 8 \\
\hline & & & \\
\hline
\end{tabular}

One major drawback of the empirical analysis is the limitation of data, which only cover the period 1999-2006. With a larger number of 5-year periods it would be feasible to consider additional variables like $\left(A_{r r}\right)^{3}$ and $\left(A_{R(r)}\right)^{3}$ to capture $S$-shaped response patterns. Another future option is to include location information about zones, bringing the analysis to a very fine level of resolution.

\section{REFERENCES}

Abdel-Rahman, H. Fujita, M. (1990), "Product Variety, Marshallian Externalities, and City Sizes", Journal of Regional Science, 30: 165-183.

Anas, A., Arnott, R. And Small, K. (1998), Urban Spatial Structure, Journal of Economic Literature, XXXVI: 1426-1464.

Andersson, M. Klaesson, J. (2006), "Growth Dynamics in a Market-Accessibility Hierarchy Does the ICT service sectors follow the overall pattern?", in The Emerging Digital Economy: Entrepreneurship, Clusters and Policy, eds. Johansson, B. Karlsson, C. Stough, R. R., Springer-Verlag, Berlin.

Baldwin, R.E. Martin, P. Ottaviano, G. (2001), "Global income divergence, trade and industrialization: the geography of growth take-offs", Journal of Economic Growth, 6:.537.

Forslund-J UM (1998), Education Intensity and Interregional Location Dynamics, in Accessibility, Trade and Locational Behaviour, ed. Reggiani A., Ashgate, Aldershot.

Forslund-J UM and Johansson B (2000), Product Vintages and Specialisation Dynamics in a Hierarchical Urban System, in Learning, Innovation and Urban Evolution, eds. Batten D.F., Bertuglia C.S., Martellato D. And Occelliu S., Kluwer Academic Pubblishers, Boston.

Fujita, M. Thisse, J-F. (2002), Economics of agglomeration: cities, industrial location, and regional growth, Cambridge University Press, Cambridge.

Glaeser, E.L., et al. (1992) Growth in Cities, Journal of Political Economy, 100:1126-1152. 
Glaeser EL (2000), The New Economics of Urban and Regional Growth, in C Gordon, G Meric and M Feldman (eds), The Oxford Handbook of Economic Geography, Oxford University Press, Oxford, pp 83-98.

Grossman G and Helpman E (1991), Innovation and Growth in the World Economy, The MIT Press, Cambridge, Mass.

Henderson JV (1988), Urban Development Theory, Fact and Illusion, Oxford University Press, Oxford.

Hohenberg, P. Lees, L. H. (1985), The Making of Urban Europe (1000-1950), Harvard Univ. Press, Cambridge.

Johansson B and Klaesson J (2007), Infrastructure, Labour Market Accessibility and Economic Development, in C Karlsson, WP Andersson, B Johansson and K Kobayashi (eds), The Management and Measurement of Infrastructure - Performance, efficiency and innovation, Edward Elgar, Cheltenham. pp 69-98

Johansson, B. Klaesson J. Olsson, M. (2003), "Commuters' non-linear response to time distances", Journal of Geographical Systems, vol. 5, 315-329.

Karlsson C. and Johansson B. (2006), Dynamics and Entrepreneurship in a Knowledge-Based Economy, in C. Karlsson, B. Johansson and R.R. Stough (eds), Entrepreneurship and Dynamics in the Knowledge Economy, Routledge, New York and London, pp 12-46

Klaesson, J. Pettersson, L. (2006), "Interdependent Urban-Rural Development in Sweden”, in Contributions to Spatial Science, eds. Charlie Karlsson, Roger Stough, Paul Cheshire, Åke Andersson, Springer Verlag, New York. Martin and Ottaviano (2001),

Rivera-Batiz F. L. (1988), "Increasing Returns, Monopolistic Competition, and Agglomeration Economies in Consumption and Production”, Regional Science and Urban Economics, 18, pp. 125-153.

Romer P (1990), Endogenous Technological Change, Journal of Political Economy, 98:71102.

Martin, P., Ottaviano, G. (1999), "Growing Locations: Industry Location in a Model of Endogenous Growth", European Economic Review, Vol. 43 pp.281-302. 